\title{
Within-Mother Estimates of the Effects of WIC on Birth Outcomes in New York City
}

\author{
Janet Currie and \\ Princeton University and NBER \\ Ishita Rajani \\ Princeton University
}

\begin{abstract}
There is a large literature suggesting that "WIC works" to improve birth outcomes. However, methodological limitations related to selection into the WIC program have left room for doubt about this conclusion. This paper uses birth records from New York City to address some limitations of the previous literature. We estimate models with mother fixed effects to control for fixed characteristics of mothers and we directly investigate the way that time-varying characteristics of mothers affect selection into the WIC program. We find that WIC is associated with reductions in low birth weight, even among full term infants, and with reductions in the probability that a child is "small for dates." These improvements are associated with a reduction in the probability that the mother gained too little weight during pregnancy. Improvements tend to be largest for first born children. We also find that women on WIC are more likely to be diagnosed with chronic conditions, and receive more intensive medical services, a finding that may reflect improved access to medical care.
\end{abstract}

There is growing acceptance of the idea that prenatal conditions can have health effects not only at the time of the birth, but lasting into adulthood (Almond and Currie, 2011). This observation leads people to question whether there are "shovel ready" policies that can be implemented to improve children's life chances? The U.S. Supplemental Nutrition Program for Women, Infants, and Children (called WIC) would seem to be ideally suited to play such a role. The program provides nutritious food, counseling, and assistance with access to medical care to pregnant women, as well as to their infants and young children. Bitler et al. (2003) show that the program serves a large fraction of pregnant women in the U.S. In fiscal year 2011, 9 million participants were served each month including 2.1 million pregnant or lactating women which can be compared to the roughly 4 million births in that year (USDA, 2012).

Yet, despite decades of research suggesting that "WIC works" in the sense that women who use WIC during pregnancy have healthier babies than other similar women, the suspicion persists that the observed positive association between WIC participation and outcomes might not be causal. ${ }^{1}$ There are two limitations to most WIC research. ${ }^{2}$ First, since women choose whether or not to participate in WIC and when to begin participating, it is possible 
that women who seek out WIC benefits are different from other observationally similar women in some unobservable way that is conducive to better birth outcomes. Despite this concern however, there has been little direct examination of the determinants of selection into the program.

Second, the longer a woman is pregnant, the longer she has to enroll in WIC. Hence, a positive correlation between WIC and length of gestation could reflect this "longer window of opportunity" effect. Moreover, since the fetus gains most of its weight in the third trimester, if women with longer gestations are more likely to sign up for WIC, then we would also expect their babies to have higher birth weights.

This paper addresses these limitations of the previous literature. Starting with a sample of all New York City births from 1994 to 2004, we focus on women with more than one singleton birth over the sample period and estimate models with mother fixed effects. In these models, the effect of WIC is identified by comparing births to the same mother, with and without WIC. This specification controls for the fact that women who use WIC may be different in some unobservable fixed respect than women who do not. It is still possible however, that time-varying characteristics of mothers are associated with both WIC participation and positive birth outcomes. Hence, we also use our data to directly investigate this type of selection and ask when mothers are most likely to use WIC in terms of these time varying characteristics?

In order to deal with the possibility of a mechanical correlation between gestation length and probability of WIC enrollment, we focus much of our attention on full term births as well as asking whether infants were "small for dates" (that is, below the $10^{\text {th }}$ percentile of national weight standards given gestational age). We also ask whether the effects of WIC are bigger for first born children. Women pregnant with their first born child may lack information about healthy practices and may not know that they are at risk for poor birth outcomes, so it is possible that WIC would have particularly large effects in this group.

We are able to document important facts about selection into the WIC program - mothers who change WIC status between births are more likely to use WIC when they are younger, unemployed, and/or unmarried. Women are also much more likely to be referred to WIC if they have had a previous poor pregnancy outcome. However, mothers are also more likely to be diagnosed with chronic hypertension or chronic diabetes when they are on WIC suggesting that WIC may improve access to medical care and/or screening for these conditions.

We find that WIC is associated with reduction in low birth weight, even among full term infants, and with reductions in the probability that a child is "small for dates." These improvements are associated with a reduction in the probability that the mother gained too little weight during pregnancy. Effects on low birth weight are concentrated in first born infants, although effects on being "small for dates" and weight gain are present for all WIC

${ }^{1}$ See Currie (2003) for a review of the older WIC literature and a discussion of its limitations. Also see Besharov and Germanis (2001) and Joyce et al. (2005) for further discussion of the limitations of the WIC literature.

${ }^{2}$ See Ludwig and Miller (2005). 
mothers. These findings suggest that WIC counselling may be particularly useful for first time mothers. We also find that women on WIC and their infants receive more intensive medical services, a finding that may reflect improved access to medical care.

\section{Background}

This section does not attempt to review all of the voluminous literature on WIC (see Currie, 2003 for a review of the older literature). Instead, we first provide an overview of the program, and then focus on studies that have attempted to address some or all of the empirical problems outlined above, and discuss how our study builds on and adds to these efforts.

WIC is a supplemental feeding program for pregnant and lactating women, infants, and children up to age 5. In addition to belonging to one of these groups, a WIC participant must either have income less than $185 \%$ of the federal poverty line, or must have Medicaid coverage (available to pregnant women with incomes less than $200 \%$ of the federal poverty line. In New York, the threshold was about $\$ 38,000$ for a family of three in 2012). Women must also be evaluated and deemed to be "at nutritional risk," though in practice this requirement does not seem to be very binding (Bitler et al., 2003).

WIC clients receive coupons or a debit card which can be used to purchase only specifically selected nutritious food items. ${ }^{3}$ In 2012, New York WIC provided food benefits worth an average of $\$ 55$ per participant per month. ${ }^{4}$ To maintain their benefits, clients are required to attend nutritional counseling and breastfeeding education sessions at regular intervals. One of the open questions about WIC is why it works (if indeed it does) given that the benefits are small relative to people's monthly food budgets. Possible answers are that the healthy WIC foods available or the nutritional counseling "nudge" people's diets in a healthier direction, or that the availability of WIC benefits helps to facilitate access to medical care.

As discussed above, most studies of WIC have difficulty dealing with possible non-random selection of women into WIC benefits. If women who enroll in WIC are, for example, more concerned about their child's health, or more motivated to change their behaviors than other women, then the measured positive effects of WIC could reflect these unobserved characteristics of mothers who enroll in the program.

In view of this concern, it is surprising that few WIC studies have estimated models with maternal fixed effects since these models offer a way to control for all observed and unobserved fixed characteristics of mothers. ${ }^{5}$ The study closest in spirit to ours is Kowaleski-Jones and Duncan (2002) who use data from the Children of the National Longitudinal Survey of Youth and estimate mother fixed effects models. Unfortunately, they had only 104 sibling pairs, and only 71 with discordant WIC participation. Nevertheless,

\footnotetext{
${ }^{3}$ As an example, the New York regulations state the following about infant cereal: "This is the only brand of cereal allowed for infants up to 12 months old: Gerber Cereal for Baby; Plain varieties only: barley, oatmeal, rice, whole wheat, or mixed; 8 and 16-ounce containers are allowed; NOT ALLOWED: Organic, extra ingredients such as DHA, fruit, formula, or added protein."

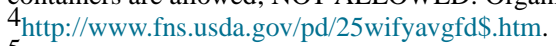

${ }^{5}$ A third study, by Hicks and Langham (1985) focuses on the beginning of the WIC program in Louisiana. They look at 19 sibling pairs in which, due to the timing of the phase in, one child received WIC in utero, and the other did not receive it until they were already a year old. They find positive effects of prenatal WIC participation on IQ and cognitive test scores.
} 
they found a statistically significant effect on birth weight (of 7 ounces) and a positive effect on temperament scores. They did not find a statistically significant effect on measures of motor and social skills.

Rossin-Slater (2012) estimates maternal fixed effects models in a study of the effects of the closure of WIC clinics in Texas. She finds that having a WIC clinic in ones zip code increases maternal weight gain and birth weight, but is also associated with more diagnoses of diabetes and hypertension during pregnancy. She speculates that this latter result might reflect better access to medical care, which in turn leads to higher diagnosis rates.

Joyce et al. (2005) also examine birth records for New York City. In order to deal with selection, they focus on relatively narrowly defined groups, e.g. Hispanic mothers with a first pregnancy, and use matching models with propensity scores. In order to deal with the mechanical correlation between gestation and the window available for WIC enrollment (i.e. the fact that longer pregnancies both allow more time to enroll and also generally result in healthier infants), they focus on birth weight conditional on gestational age. Joyce et al. (2008) examine data from nine states and ask whether earlier initiation of WIC is associated with better maternal behaviors and better birth outcomes. Both papers argue that a positive association between WIC and gestation is suspect for the reasons given above. Both of these papers do however find positive effects of WIC on infant outcomes, though they argue that the effects are smaller than those found in the previous literature.

Bitler and Currie (2005) and Figlio et al. (2009) also attempt to deal with selection by narrowing the comparison group. Bitler and Currie focus on a large national sample of mothers whose births were paid for by Medicaid (so that all of the mothers were adjunctively eligible for WIC). Figlio et al. use administrative data from Florida and focus on families in a narrow income band around the WIC eligibility threshold. They also use a tightening of WIC income documentation requirements to try to identify the effect of WIC receipt. Using this design, they find a strong effect of WIC on the incidence of low birth weight (birth weight less than 2500 grams) even though, as they point out, the marginal mother eligible for WIC may benefit less than infra-marginal recipients.

Hoynes et al. (2011) focus on the roll out of the WIC program in the late 1970s in a difference-in-differences framework and show that in counties that adopted WIC, the incidence of lower birth weight fell. However, arguably the factors affecting the nutritional status of pregnant women could be quite different today than they were in the 1970s, given sharp declines in the relative price of food over time, as well as large increases in obesity.

This brief review indicates that our study is one of very few to examine the effects of WIC in models that control for mother fixed effects. It is also the first large scale analysis of selection into WIC, and the first to examine effects of WIC by birth order. The exploration of within-mother selection into the WIC program and of the effects of WIC on firstborns is of independent interest and sheds light on the way that "WIC works." 


\section{Methods}

In order to investigate the way that time-varying maternal characteristics are associated with participation in the WIC program, we estimate models of the form:

$$
Z_{\mathrm{it}}=a_{\mathrm{i}}+a_{1} \text { WIC }+a_{2} X_{\mathrm{it}}+a_{3} \text { Month }+a_{4} \text { Year }+a_{5} \text { Neighborhood }+e_{\mathrm{it}},
$$

where $\mathrm{Z}_{\mathrm{it}}$ is one of a vector of maternal characteristics including pre-pregnancy weight, whether she has chronic diabetes, whether she has chronic hypertension, age, whether she smoked at all during the pregnancy, whether she was employed, whether she was married, and whether the mother had a previous poor pregnancy outcome (the later outcome can only be examined in mothers who had a previous birth that is included in our data set). The vector $a_{\mathrm{i}}$ represents a fixed effect for each mother. The vector $X_{\mathrm{it}}$ includes time varying characteristics of the mother and child which might be associated with selection into the program including child gender, maternal education $(<12,12,13-15,16+$, missing), parity $(1,2,3,4+$, missing), and maternal age $(<20,20-24,25-29,30-34,35+$, missing). (Note that these age dummies are omitted when the dependent variable in question is maternal age). The coefficient of interest is $a_{1}$ which measures the correlation between changes in the time varying dependent variables and changes in WIC status between pregnancies.

We also include dummy variables for each month and year of birth in order to control for time trends in these variables, and for maternal neighborhoods within New York City, since neighborhood is an important indicator of economic and social status. ${ }^{6}$ Finally, $e_{\mathrm{it}}$ represents a random error term.

Our object in estimating (1) is to determine whether time varying characteristics of mothers are systematically related to WIC enrollment. If we find that a mother is more likely to be on WIC when she has a dangerous chronic condition that complicates her pregnancy, then we should take this information into account when interpreting the within-mother estimates of the effects of WIC participation on birth outcomes. For example, chronic diabetes is associated both with a higher risk of a small and preterm baby, and with a higher risk of a very large baby with a complicated delivery. Children of diabetic mothers also have a higher incidence of congenital anomalies and so may be at higher risk of negative developmental outcomes (Nold et al., 2004). If mothers diagnosed with diabetes were more likely to be referred to WIC, then this type of selection could bias the estimated effect of WIC on birth outcomes.

Turning to outcomes of pregnancy that we might expect to be affected by the WIC program, we estimate models of the form:

$$
Y_{\mathrm{it}}=a_{\mathrm{i}}+a_{2} \text { WIC }_{\mathrm{it}}+a_{3} Z^{\prime}{ }_{\mathrm{it}}+a_{4} X_{\mathrm{it}}+a_{5} \text { Month }+a_{6} \text { Year }+a_{7} \text { Neighborhood }+e_{\mathrm{it}},
$$

\footnotetext{
${ }^{6}$ Neighborhoods are defined by the Department of City Planning and are at the level of areas such as "Brighton Beach," "Clinton Hill," or "Hunts Point." See http://www.nyc.gov/html/dcp/html/bytes/dwn_npa.shtml.
} 
where $Y_{\text {it }}$ is a vector of outcomes including whether the baby is low birth weight, whether the baby is small-for-dates, whether the baby had a low APGAR score, whether the baby was preterm (gestation less than 38 weeks), whether the baby was admitted to the neo-natal intensive care unit (NICU), and whether there were complications of labor and delivery; WIC is an indicator for whether the mother used WIC during the pregnancy, and Z' includes the following time-varying characteristics of the mother: pre-pregnancy weight, whether she has chronic diabetes, whether she has chronic hypertension, whether she smoked at all during the pregnancy, whether she was employed, whether she was married. The other variables are defined as in (1) except that $X_{\text {it }}$ includes one additional variable which is whether the information about the father is missing from the birth certificate. This variable can be viewed as an indicator of father involvement.

Finally, we also estimate models that allow the effects of WIC to differ for first born children. There are two reasons why this exercise is of interest. One is that first born children may be more affected by WIC because their mothers do not know what to expect and so the guidance offered by WIC might be of particular value. Second, as we show below, mothers who have had a previous poor pregnancy outcome (such as an infant death) are more likely to use WIC in the current pregnancy. Failure to control for this negative selection into WIC could result in downward bias in the estimated effect on infant health; therefore it is of interest to compare the effects of WIC on firstborns with the effects of WIC for subsequent pregnancies. These models take the form:

$Y_{\mathrm{it}}=a_{\mathrm{i}}+a_{2} W I C_{\mathrm{it}}+a_{3} W I C_{\mathrm{it}} *$ Firstborn $+a_{4} Z^{\prime}{ }_{\mathrm{it}}+a_{5} X_{\mathrm{it}}+a_{6}$ Month $+a_{7}$ Year $+a_{8}$ Neighborhood $+e_{\mathrm{it}}$,

Where Firstborn is an indicator equal to one if the child is the mother's first born, and all other variables are defined as described above. In this regression, $a_{2}$ is the estimated effect of WIC on all children, and a significant value for $a_{3}$ indicates that the effect is different for firstborns.

We conduct our analyses for all mothers, and for subsamples of mothers defined by Medicaid coverage, education less than 12 years, and by race. We examine these subsamples largely for the sake of comparability with previous research. Some previous analyses have found larger effects of WIC among African-American mothers. As discussed above, several previous analyses have confined their attention to mothers with Medicaid coverage. Finally, one might think that the counseling aspect of WIC could be particularly valuable to less educated mothers, so we also examine this group separately.

\section{Data}

The data for this study come from the New York City Department of Health and Mental Hygiene's Vital Statistics Natality system for collecting and recording information from the certificate of live birth. Data for these certificates come from two worksheets. One is 
completed by the mother and asks information about her circumstances and behaviors (such as marital status, smoking during pregnancy, and pre-pregnancy weight). The other worksheet is completed by the medical facility where the birth takes place using medical records. This worksheet includes information about prenatal care visits, risk factors for the pregnancy, complications of labor and delivery, and newborn health. We start with all live singleton births in New York City between 1994 and 2004, which yields approximately 1.2 million records.

Table 1 provides an overview of our data. In the full sample, $42 \%$ of the births were to mothers who used WIC at some point during pregnancy. The second column of Table 1 shows means for those children with a sibling in the sample, roughly $45 \%$ of the sample births. The remaining two columns focus on this subsample of sibling births, and divide them into births in which the mother used WIC and births in which she did not. Focusing on these last two columns, we can see that the incidence of negative outcomes is generally higher for the WIC infants: They are more likely to be low birth weight, small for dates, to have low APGAR scores (a measure of the health of the infant at birth that is taken out of 10), and to be premature. ${ }^{7}$ Strikingly, the WIC infants are almost twice as likely as the nonWIC infants to end up in the neo-natal intensive care unit (NICU).

Of course much of the gap between the WIC infants and the non-WIC infants is likely to be due to differing maternal characteristics. Table 1 indicates that mothers of the WIC infants were much less educated (a mean difference of more than two years), younger, half as likely to be married, twice as likely to be missing the father's information on the birth certificate (an indicator of paternal involvement), and more likely to smoke. They also have higher incidence of chronic diabetes and chronic hypertension, are less likely to receive prenatal care in the first trimester of pregnancy, and are almost entirely dependent on Medicaid coverage. The WIC mothers are a little heavier on average before the pregnancy but less likely to have gained an adequate amount of weight during pregnancy. ${ }^{8}$ They are also more likely to have complications of labor and delivery. Finally WIC mothers who have already had one child are more likely to have had a poor outcome in a previous pregnancy. We define a poor outcome to include: A previous infant death (very rare), a prior child admitted to the NICU, a previous infant who was premature, or a previous low birth weight infant. These stark differences between the circumstances of WIC infants and non-WIC infants illustrate the importance of adequately controlling for the characteristics of WIC mothers when evaluating the effects of the program on outcomes.

\section{Results}

Table 2 examines the relationship between maternal characteristics and participation in the WIC program using mother fixed effects models. Therefore, these estimates rely on the sample of mothers who used WIC for one pregnancy but did not use it for another to tell us when they were relatively more likely to use WIC. The table shows estimates for all births,

\footnotetext{
${ }^{7}$ Birth weights $<700 \mathrm{~g}$ and $>7000 \mathrm{~g}$ have been recoded as missing. The dummy for gestation $<37$ weeks has been recoded as missing for gestation $<20$ weeks and $>45$ weeks.

${ }^{8}$ Pre-pregnancy weight has missing values in the data and in addition values $<91$ pounds have been coded as missing when this is used as an outcome.
} 
full term births only, and for different groups of mothers defined by insurance status, race, and education.

The first three columns of Table 2 show that mothers tend to use WIC when they are not employed, when they are unmarried, and when they are younger. These differences exist for all the groups we examine, except that there is no difference in age among less educated mothers. All but African-American mothers have lower pre-pregnancy weights when they go on WIC, whereas African-American mothers, who are the highest mean weight group, are more likely to use WIC when they are relatively heavy. Together these results suggest a scenario in which mothers use WIC (and smoke) when they are relatively young, unemployed, and unmarried. As they grow older they may be more likely to quit smoking, become employed, and/or marry and they become less likely to use WIC.

It is surprising then that we also find that women are more likely to have chronic diabetes and chronic hypertension when they are on WIC since these are conditions that increase in prevalence with age. An examination of the subgroups shows that this relationship is strongest among African-American women. We think that, consistent with Rossin-Slater's interpretation of her results from Texas, women on WIC may be more likely to be properly diagnosed and treated if they have chronic diabetes or hypertension, so that these conditions are more likely to be subsequently recorded on the birth certificate. Consistent with this interpretation, Table 2 also shows that women on WIC are more likely to get prenatal care in the first trimester. ${ }^{9}$

The last column of Table 2 shows that in the subset of women who have already had a birth that we can observe, women who had a previous poor pregnancy outcome are much more likely to be on WIC. This finding could reflect women being more motivated to try to improve their pregnancy outcomes, or a pattern of referring women with poor prior outcomes to WIC.

Changes in these time varying maternal characteristics are likely to bias estimated WIC effects if they are not controlled. The fact that women use WIC when they are relatively disadvantaged (young, unmarried, unemployed) would be expected to bias estimated effects of WIC downwards in the absence of controls for these variables. Similarly, if women who know that they are at risk of future poor outcomes are more likely to use WIC, then estimates that do not take this selection into account would understate the benefits of WIC. If women on WIC are really more likely to have chronic diabetes and/or hypertension, then this would also tend to be reflected in poorer birth outcomes. However, as discussed above, it is possible that this pattern reflects differential reporting of chronic conditions for women on WIC rather than an actual increased prevalence of these conditions.

Table 3 shows estimated effects of WIC on infant outcomes. The estimates in this table and in Table 4 are based on equation (2) and include observable time-varying characteristics associated with selection into the WIC program: Maternal age, employment, marital status, smoking during pregnancy, pre-pregnancy weight, ${ }^{10}$ chronic diabetes, chronic hypertension,

${ }^{9}$ An alternative possibility is that women on WIC are being unnecessarily diagnosed with these conditions and treated, but we think this is unlikely given the clear diagnostic criterion and lack of debate about appropriate treatment for high blood pressure and diabetes. 
and whether women received prenatal care in the first trimester. We have also estimated these models excluding the prenatal care variable, and find that this exclusion has little effect on the estimates.

Since we only observe previous poor pregnancy outcomes for a subset of women in our data set, we have not controlled for that variable here. Below, we show estimates which allow the effect of WIC to vary for first born children and other children. These estimates will shed light on the potential importance of any information that mothers may have gained from previous pregnancies, since no mother has such information for first births.

Column 1 in the first panel suggests that consistent with the vast majority of WIC studies, WIC participation is estimated to have a negative effect on the incidence of birth weight less than 2500 grams: The coefficient in the first row and column of .0041 can be compared to the fraction low birth weight which is also given in the table and thus implies a $6.12 \%$ reduction in the overall probability of low birth weight. However, it is possible that some of the estimated "effect" is driven by the mechanical correlation between gestation and the probability of enrollment in WIC (due to having a longer time period in which to enroll) that was discussed earlier. Column 2 suggests that WIC has a large effect on prematurity, reducing it by $10.05 \%$ which again, may reflect this mechanical correlation. Hence, column 3 shows estimates of whether the baby was "small for dates." Conditioning on gestation length in this way suggests that WIC reduces the overall probability of a small-for-dates baby by $2.92 \%$. This smaller effect suggests that some of the estimated WIC effect in low birth weight and prematurity is indeed due to the mechanical relationships between gestation length, a longer window in which to enroll in WIC, and positive birth outcomes. However, the significant effect on whether the baby is small-for-dates indicates that WIC has a significant effect even conditional on gestation. The fourth column provides some evidence regarding potential mechanisms, suggesting that the probability of low weight gain during pregnancy (defined as weight gain less than $6 \mathrm{~kg}$ ) is significantly reduced by participation in WIC, falling by $9.18 \%$.

Columns 5 and 6 examine complications of labor and delivery, and the probability that the infant was admitted to the NICU, respectively. Surprisingly, the estimated effect of WIC is significantly positive in both cases, indicating that receiving WIC prenatally increases the probability of these outcomes. Since not even the harshest critics of WIC have suggested that it actually harms women and children, it seems likely that these estimates reflect improved access to medical care during and after the birth among WIC recipients rather than a causal effect of prenatal nutritional assistance or counseling.

The second panel of Table 3 shows estimates for mothers who were covered by Medicaid in both pregnancies. The estimated effects of WIC are almost identical to those in the full sample of mothers for all the outcomes examined. Hence, it appears that although Medicaid participants are adjunctively eligible for WIC, WIC participation is not simply a proxy for Medicaid coverage. Hence any improvements in access to medical care that are associated

\footnotetext{
${ }^{10}$ When used as a control, indicators for the following categories of pre-pregnancy weight have been included: missing, $>90-<110$ pounds, $110-<120$ pounds, $120-<135$ pounds, $135-<159$ pounds, $159-<185$ pounds, and $>=185$ pounds.
} 
with WIC are not coming solely through the provision of health insurance, but through some other channel. The estimates for mothers with less than 12 years of education are very similar to those for Medicaid covered mothers.

Turning to the estimates by race, the effect on "small for dates," which is the most reliable measure of infant health in this table, is about one third larger in terms of the point estimate for African-Americans, but is not precisely estimated given the smaller subsample. The effect of WIC on the probability that the mother gained too little weight is also negative and about a third larger than for the sample as a whole. This estimate is statistically significant. Thus, the table provides some support for the hypothesis that the effects of WIC are larger for African-American women than for other groups.

Aside from the regressions using "small-for-dates" as the dependent variable, the estimates in Table 3 are potentially colored by the mechanical correlation between gestation and WIC enrollment that has been discussed. Hence, Table 4 repeats the same analyses on the subset of infants who are full term, that is, born after 37 weeks gestation. Perhaps surprisingly, these estimates are similar to those in Table 3, and in some cases are larger. For example, in this sample WIC is estimated to reduce the probability that an infant is small for dates by $4.88 \%$. Turning to the subsamples, the effect is significant for Medicaid mothers and less educated mothers. When we look by race, the impact of WIC on low birth weight is over twice as high for African-Americans as for other mothers. The effects are also larger for African-Americans in the models of low weight gain, small-for-dates, and NICU use.

Table 5 shows estimates from model (3), which allows the effect of WIC to be different for first born children than for children of higher birth order. Columns 2 through 5 suggest that the estimates are, for the most part, quite similar to Table 4. There is no significant difference in the effect of WIC on being small for dates between firstborn and other children: Both groups of children are $4.81 \%$ less likely to be small for dates compared to siblings who did not receive WIC in utero. The effects of WIC on low weight gain are somewhat larger for firstborns, but statistically significant for all children.

Moreover, there appear to be significant effects of WIC on complications of labor and delivery and the probability of NICU use for most children, including first born children. The sole exception is among African-American mothers. In this group, WIC has no significant effect on complications of labor and delivery, and has a much weaker effect on NICU use among first borns than among other children. Recall, that we presented some evidence earlier suggesting that women with previous poor pregnancy outcomes are referred to WIC. Since mothers of first born children have not had previous poor pregnancy outcomes, the effect of WIC on the use of these medical services cannot be driven by such referrals in this group. Stronger effects of WIC on the utilization of medical care among later born African-American children are consistent with such a pattern of referrals, however.

One result that is quite different for firstborns than for other children appears in column (1). Only firstborn children have significantly lower probabilities of being low birth weight when they received WIC. Moreover, WIC is estimated to reduce the probability of low birth weight in this group by a third. 


\section{Discussion and Conclusions}

This paper presents the first large-sample mother fixed effects estimates of the effects of WIC on infant outcomes and use of health care. Since fixed effects estimates only control for fixed characteristics of mothers, we also pay careful attention to time-varying factors associated with within-mother changes in prenatal WIC participation. The portrait that emerges is one in which young, unmarried, unemployed women use WIC initially, but are less likely to use it for subsequent pregnancies in which they are older and married or employed. These results also show that investigation of the determinants of WIC participation is useful when interpreting its estimated effects.

The evidence suggests that women receive more intensive medical care when they are on WIC, at least in New York City. Women are more likely to be diagnosed with chronic diabetes and hypertension when they are on WIC despite being younger; they are more likely to be diagnosed with complications of labor and delivery; and their infants are more likely than non-WIC siblings to spend any time in the NICU. These results contrast with earlier estimates (e.g. Bitler and Currie, 2005) which suggested that WIC resulted in immediate cost savings due to reductions in use of medical care among women and newborns. Moreover, even firstborns are more likely to be diagnosed with complications of labor and delivery and to use the NICU if their mothers were on WIC during pregnancy, which suggests that the overall effect cannot be an artifact of women with poor previous pregnancy outcomes being referred to WIC, though it is possible that the pattern among African-Americans reflects such a referral pattern.

We also find, consistent with most previous WIC studies, that WIC participation has positive effects on infant health: WIC infants are less 5.6\% likely to be low birth weight and $4.9 \%$ less likely to be small for dates (even when we only look at full term infants). However, consistent with the critiques of Joyce et al. $(2005,2008)$ and others, these estimates are quite a bit smaller than many that are in the literature. ${ }^{11}$ An exception is among firstborns, where WIC reduces the probability of low birth weight by a third. Thus, the results suggest that WIC promotes weight gain and reduces the probability that a child is "small for dates" among most women, but has especially strong effects on women delivering for the first time.

One possible caveat to the current study is that the estimated effects may actually be too small. Measurement error that was independent of WIC status, which is self-reported by the mother, could bias the estimated coefficients towards zero. Also, it is not clear that one should expect the estimated effects of WIC to be the same in every time and place as they must depend on the average health and nutritional status of women in the program, the composition of program benefits, and the medical and nutritional benefits available outside the WIC program.

In summary, we find that infants whose mothers used WIC during pregnancy are healthier than siblings born when the mothers were not using WIC. Some of the effect seems to come through a reduced probability of low weight gain during pregnancy, which is reflected in

\footnotetext{
${ }^{11}$ For example, Bitler and Currie (2005) find a $29 \%$ reduction in the incidence of low birth weight. It may be that the effects of WIC are quite different in different populations, depending in part on their underlying nutritional status and access to medical care.
} 
heavier babies and a lower incidence of "small-for-dates" babies. WIC mothers in our sample were also heavier users of medical care, and were more likely to be diagnosed with chronic conditions (probably reflecting this more intensive use of care). We believe that mother fixed effects techniques applied to richer administrative data (such as future mergers of educational records with birth records) could yield even greater insights into the longterm benefits of WIC.

\section{Acknowledgments}

The authors would like to thank Katherine McVeigh, Melissa Pfeiffer, Maushumi Mavinkurve, Jisen Ho, Meredith Slopen and Slavenka Sedlar for their roles in making the data available. Thu Vu provided excellent programming assistance.

Funded by NIH. Grant Number: P2C HD047879

\section{References}

Almond, Douglas, Currie, Janet. Human Capital Development Before Age Five. In: Ashenfelter, Orley, Card, David, editors. Handbook of Labor Economics. 2011. p. 1315-1486.

Besharov, Douglas, Germanis, P. Rethinking WIC: An Evaluation of the Women, Infants, and Children Program. American Enterprise Institute; Washington D.C: 2001.

Bitler, Marianne, Currie, Janet. Does WIC work? The Effects of WIC on Pregnancy and Birth Outcomes. Journal of Policy Analysis and Management. 2005; 24(\#1):73-91. [PubMed: 15584177]

Bitler, Marianne, Currie, Janet, Scholz, John Karl. WIC Eligibility and Participation. Journal of Human Resources. 2003:1139-1179.

Figlio, David, Hamersma, Sarah, Roth, Jeffrey. Does Prenatal WIC Participation Improve Birth Outcomes? New Evidence from Florida. Journal of Public Economics. 2009; 93(1-2):235-245.

Hoynes, Hilary, Page, Marianne, Stevens, Anne. Can Targeted Transfers Improve Birth Outcomes? Evidence from the Introduction of the WIC Program. Journal of Public Economics. 2011:95.

Joyce, Theodore, Gibson, Diane, Colman, Silvie. The Changing Association Between Prenatal Participation in WIC and birth Outcomes in New York City. Journal of Policy Analysis and Management. 2005; 24(\#4)

Joyce, Theodore, Racine, Andrew, Yunzal-Butler, Cristina. Reassessing the WIC Effect: Evidence from the Pregnancy Nutrition Surveillance System. Journal of Policy Analysis and Management. 2008; 25(\#2):277-303.

Kowaleski-Jones, Lori, Duncan, Greg. Effects of Participation in the WIC Program on Birthweight: Evidence from the National Longitudinal Survey of Youth. American Journal of Public Health. 2002; 92(\#5):799. [PubMed: 11988450]

Ludwig, Jens, Miller, M. Interpreting the WIC Debate. Journal of Policy Analysis and Management. 2005; 24(\#4):691-701. [PubMed: 16201055]

Nold, Joan, Georgieff, Michael. Infants of Diabetic Mothers. Pediatric Clinics of North America. 2004; 51:619-637. [PubMed: 15157588]

Rossin-Slater, Maya. WIC in Your Neighborhood: New Evidence on the Impacts of Geographic Access to Clinics. Journal of Public Economics. 2013 


\section{을 \\ 졸}

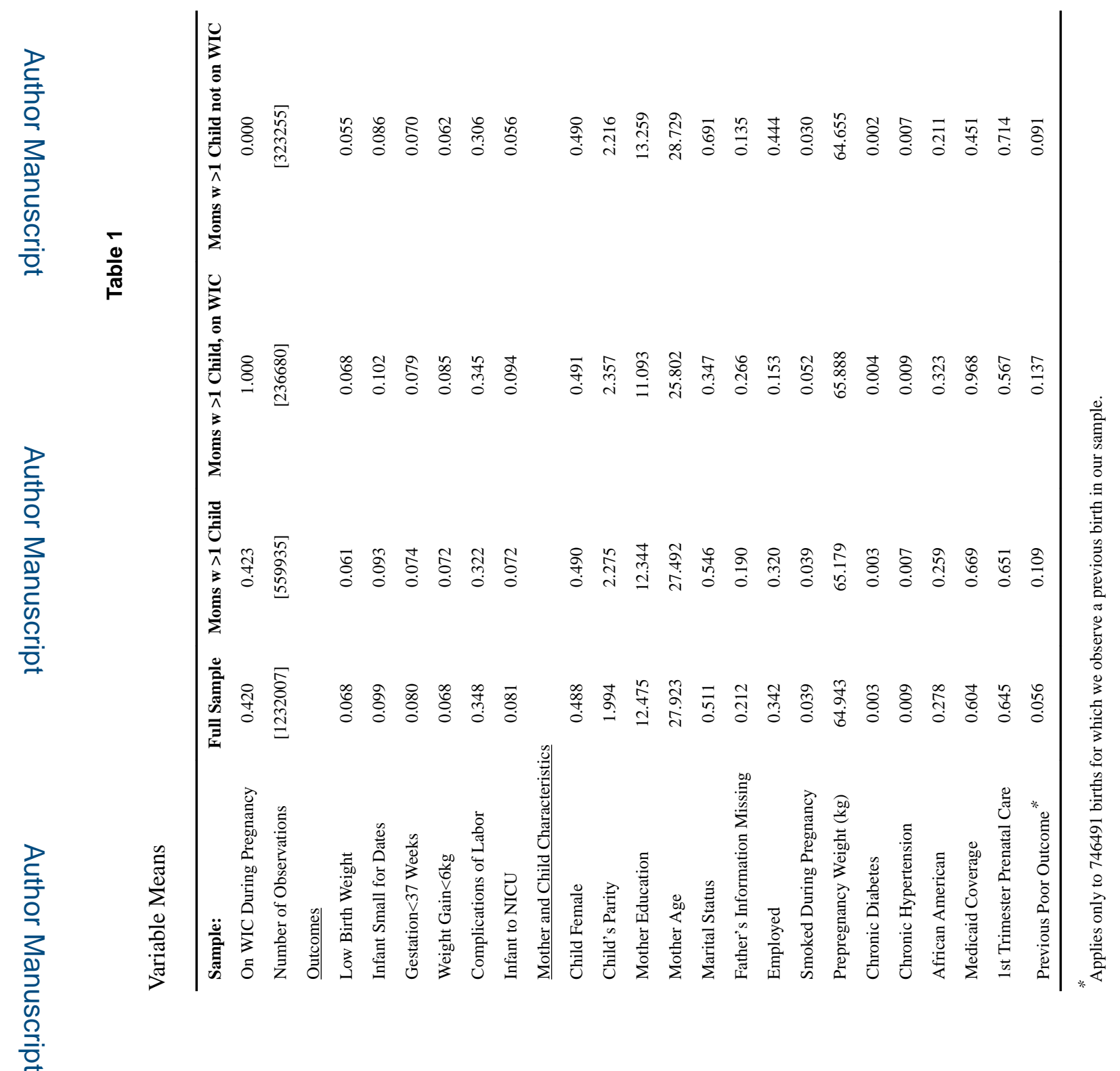

Econ Inq. Author manuscript; available in PMC 2017 May 10. 


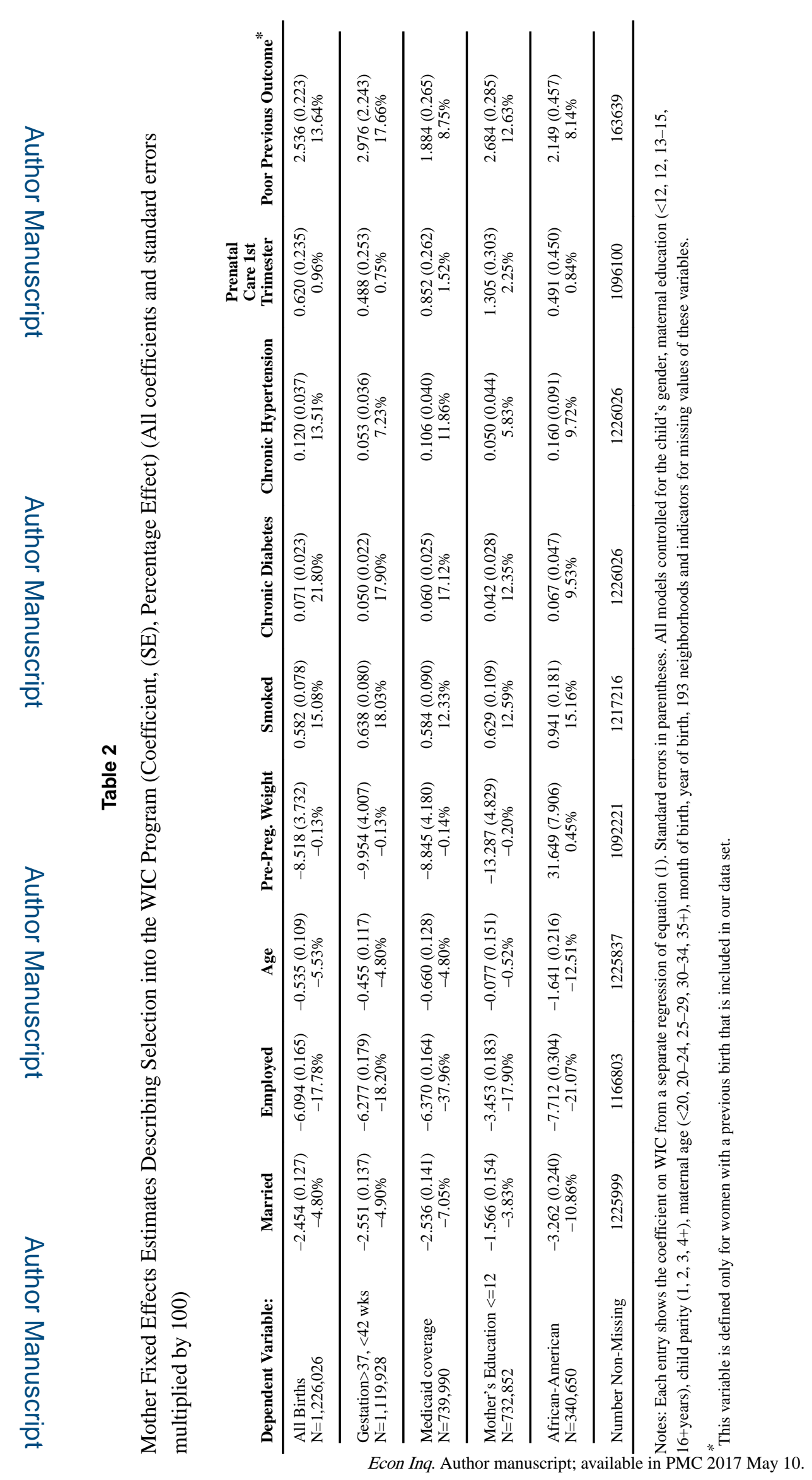




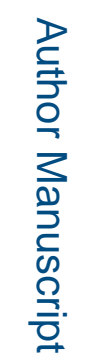

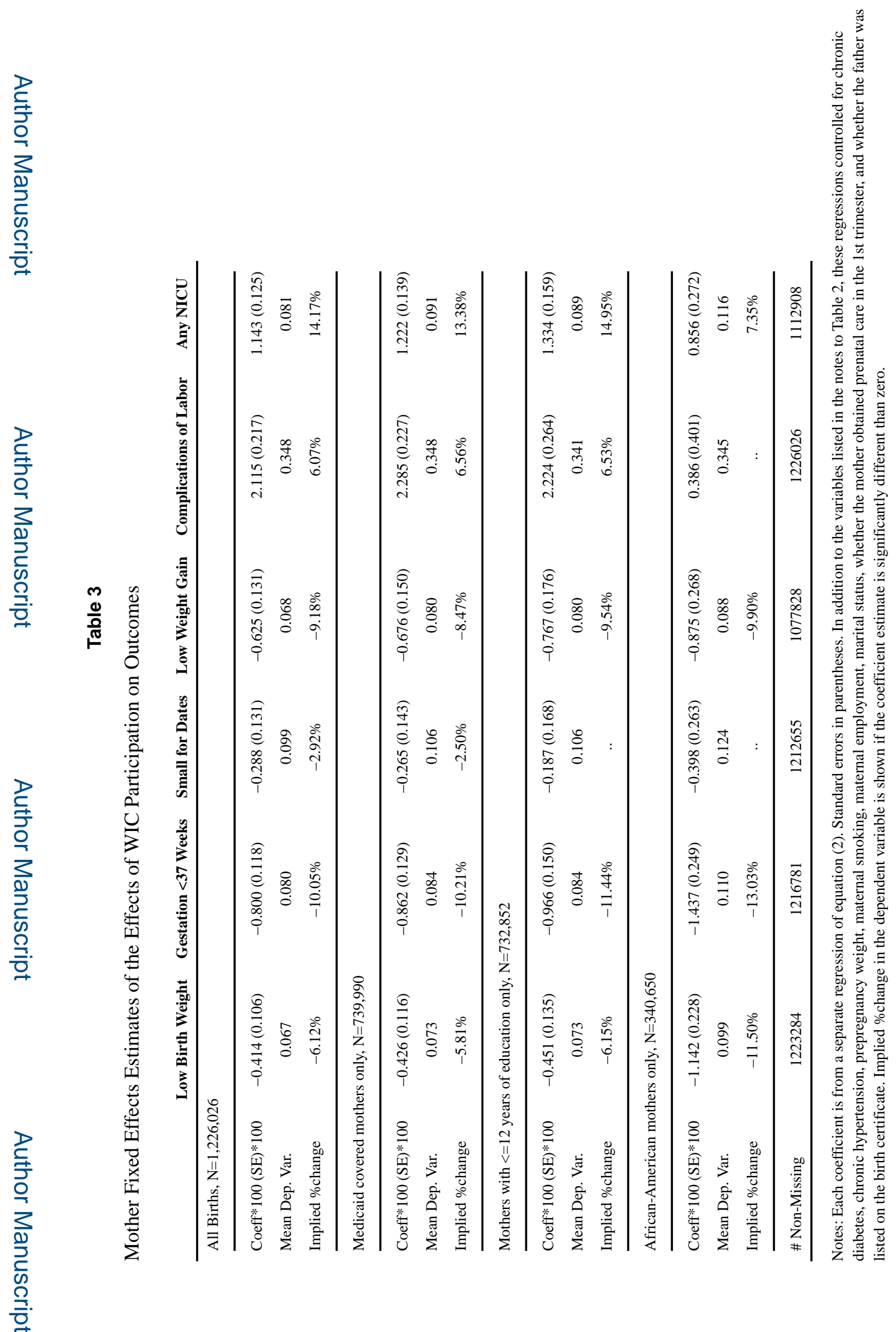

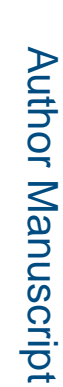

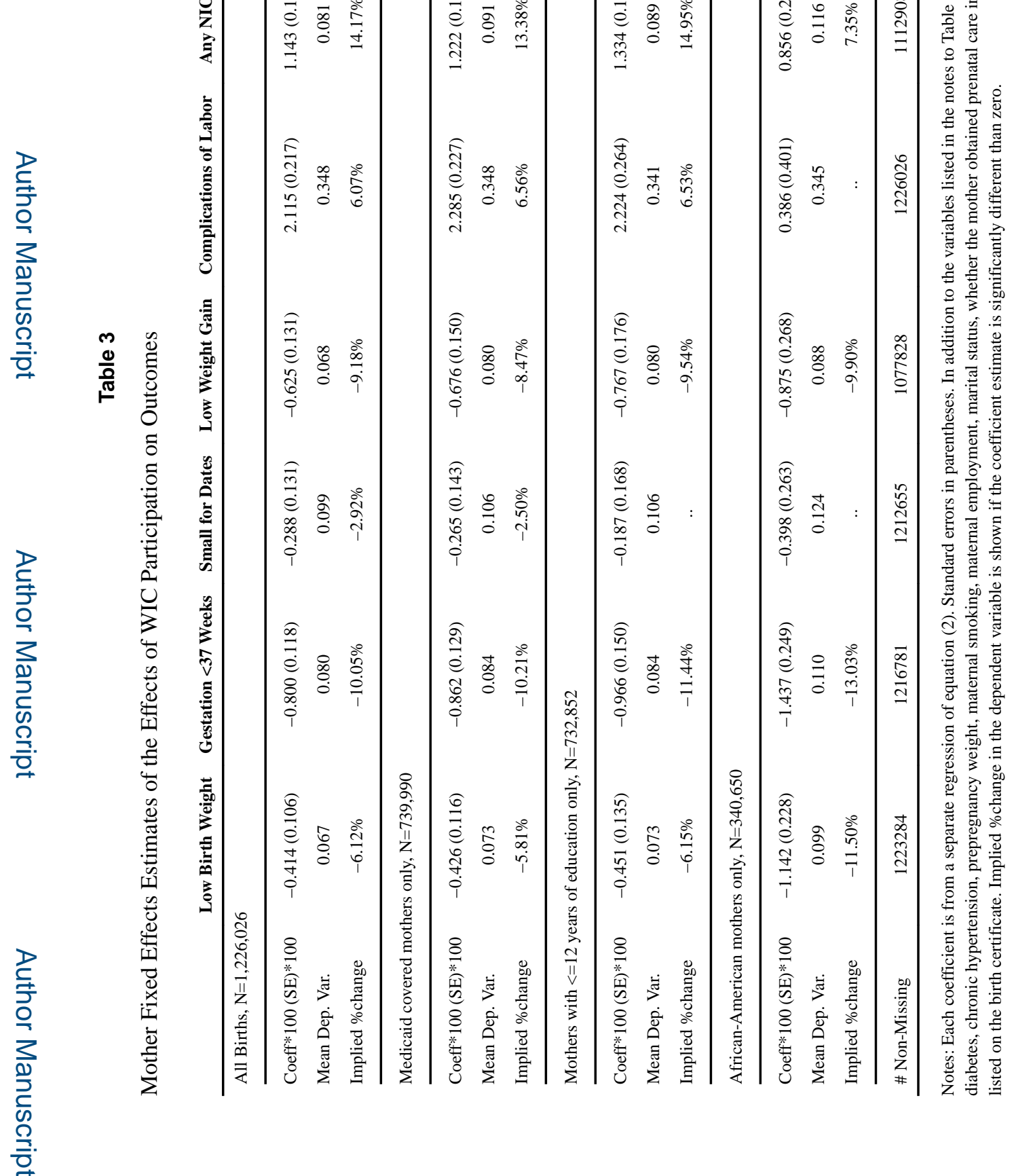

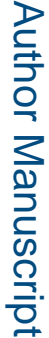

Econ Inq. Author manuscript; available in PMC 2017 May 10. 


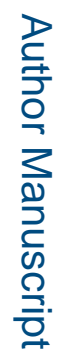

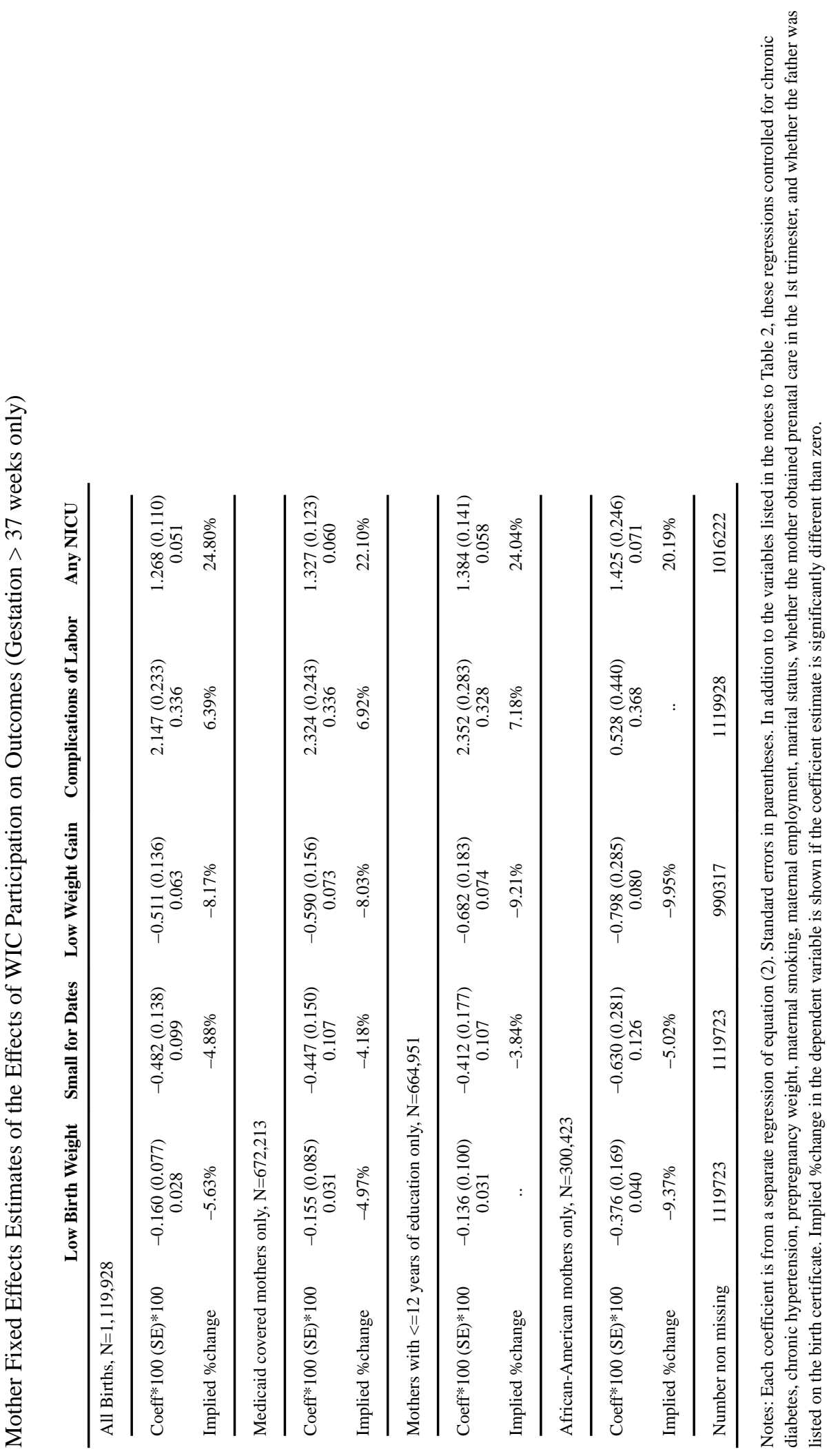

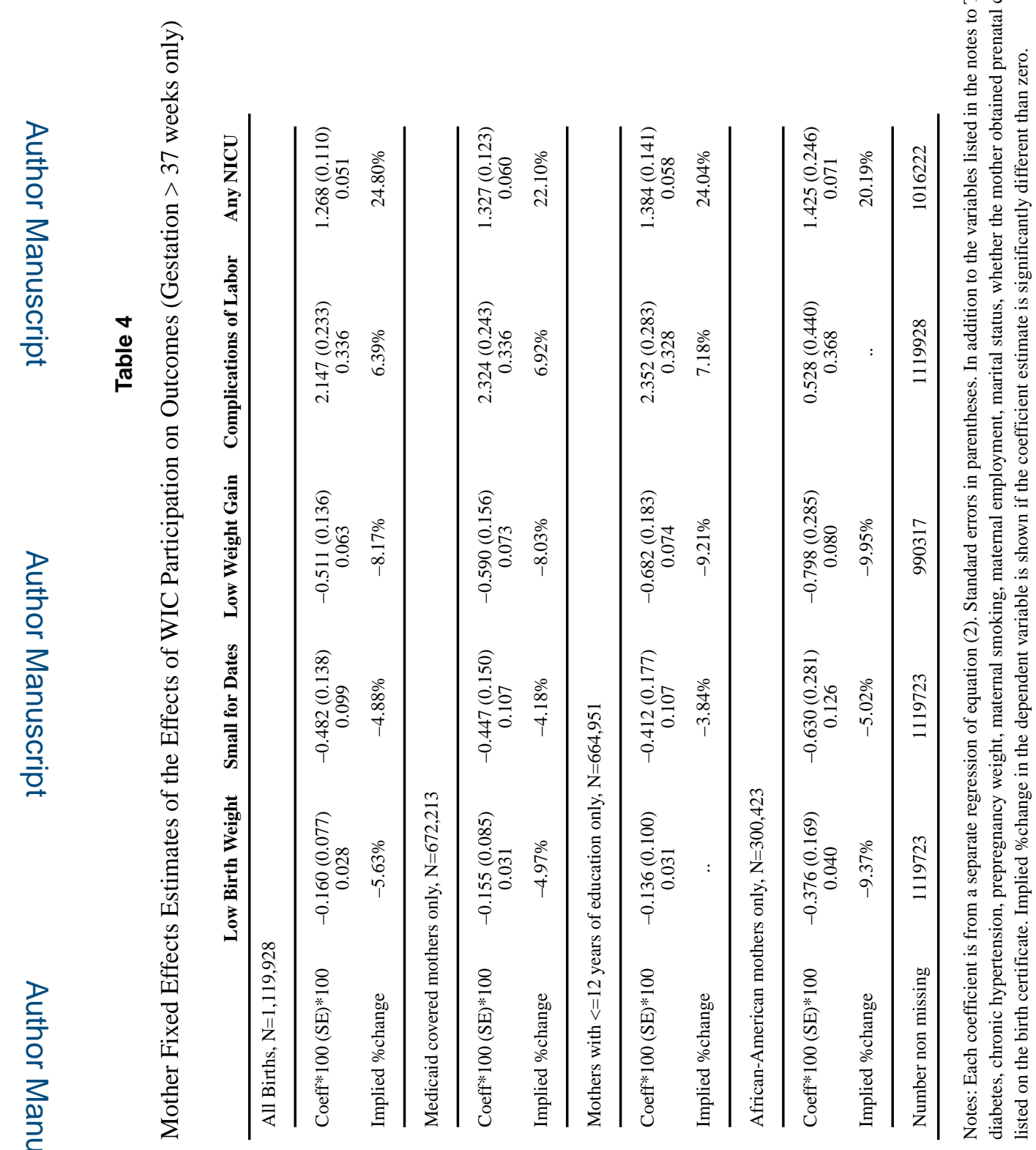




\section{롤 \\ 交}

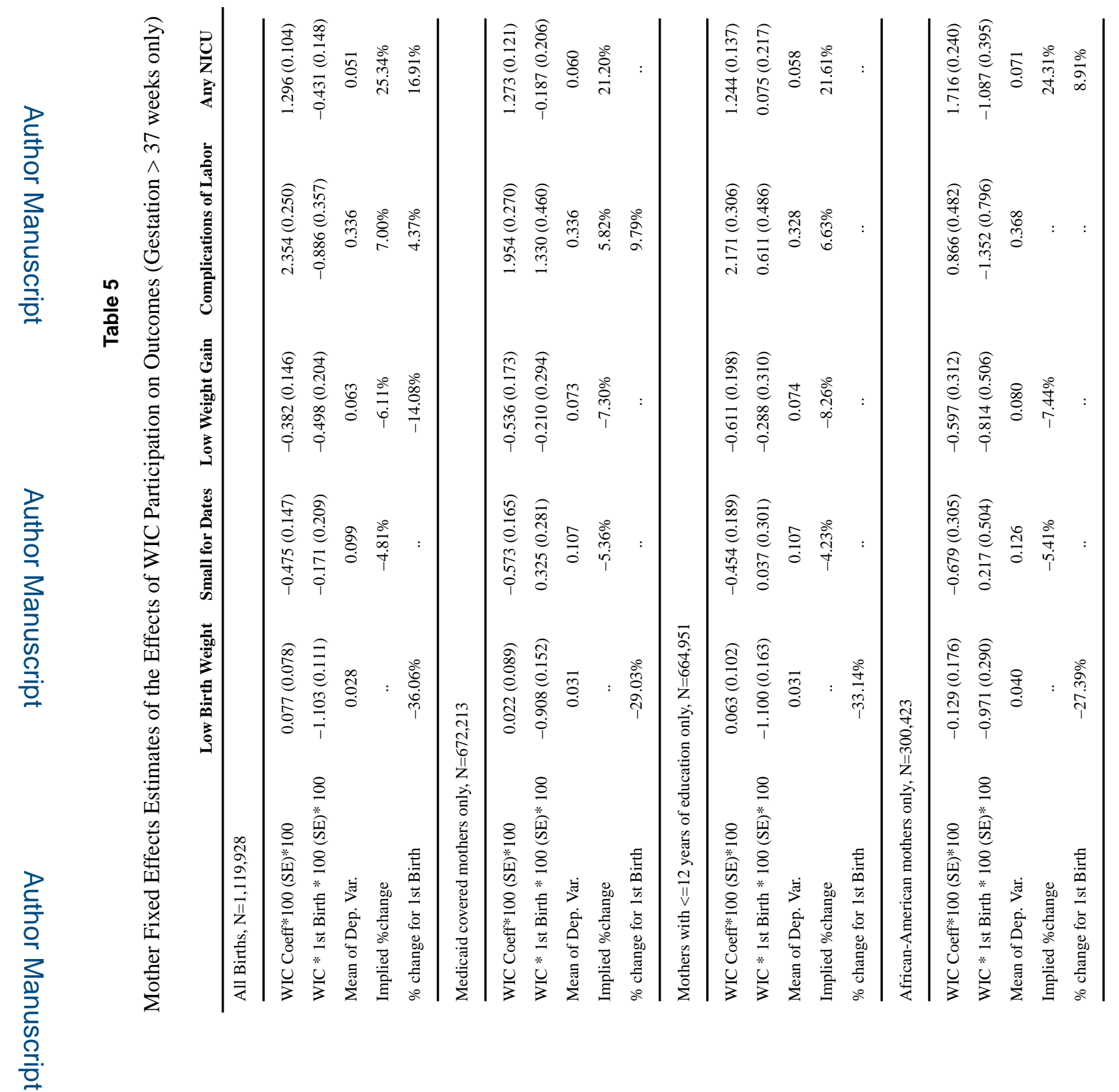

Econ Inq. Author manuscript; available in PMC 2017 May 10. 


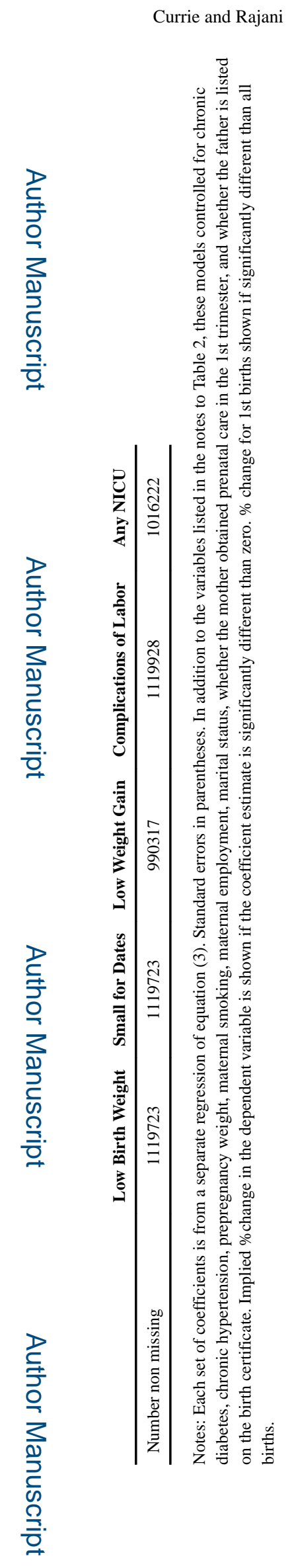

Page 18 